\title{
A Patient of Advanced NSCLC with a New EGFR Exon 19 Insertion Mutation and its Response to EGFR-TKls
}

\author{
Ning Zhu1, Caixia Dong1, Shanshan Weng1 and Ying Yuan1,2 \\ IDepartment of Medical Oncology, The Second Affiliated Hospital, Zhejiang University School of Medicine, Zhejiang Province, China \\ ${ }^{2}$ Cancer Institute, The Second Affiliated Hospital, Zhejiang University School of Medicine, Zhejiang Province, China
}

\begin{abstract}
Epidermal growth factor receptor tyrosine kinase inhibitors (EGFR-TKIs) are the standard therapy for patients with advanced non-small-cell lung cancer (NSCLC) harbouring common EGFR mutations. However, about $10 \%$ of EGFR mutations are uncommon mutations and their response to EGFR-TKIs remains unclear. The present case reports a 75-year, female patient with advanced NSCLC, presenting with a new subtype of EGFR exon 19 insertion mutation (IPVAIL insertion), who showed obvious symptom improvement after EGFR-TKls treatment but a relatively short time of progression-free survival (PFS) and succumbed to tumor 133 days (4.4 months) after diagnosis. In conclusion, patients harbouring new subtype of EGFR exon 19 insertion mutations, IPVAIL insertion may have a poor prognosis. Further experiences are required to characterise these uncommon mutations.
\end{abstract}

Key Words: Gefitinib, afatinib, exon 19 mutation, non-small cell lung cancer, epidermal growth factor receptor.

How to cite this article: Zhu N, Dong C, Weng S, Yuan Y. A patient of advanced NSCLC with a new EGFR exon 19 insertion mutation and its response to EGFR-TKIs. J Coll Physicians Surg Pak 2019; 29 (Supplement 2):S126-S128.

\section{INTRODUCTION}

Non-small-cell lung cancer (NSCLC) is the most common type of all lung cancers (about $85 \%$ ). ${ }^{1}$ Activating EGFR mutations are main oncogenic drivers in NSCLC. With the application of the next-generation sequencing (NGS), more and more uncommon EGFR mutations are being detected. However, EGFR exon 19 insertions, first reported by $\mathrm{He}$ et al. in 2012, are very rare (about $0.5 \%$ of all EGFR mutations). ${ }^{2}$ Their sensitivity to EGFR-TKIs in patients with NSCLC is uncertain. Here, we report a case of a patient with advanced NSCLC harbouring IPVAIL insertion, a new subtype never reported before and its response to EGFR-TKIs.

\section{CASE REPORT}

In August 2017, a 75-year Chinese female patient presented at our hospital with a one-year history of repeated epigastria discomfort and pain in her lower back. The symptoms of appetite and weight loss aggravated in recent three months. The eastern cooperative oncology group (ECOG) performance status score of the patient was one. ${ }^{3} \mathrm{~A}$ serum tumor markers test panel was used for tumor screening and showed the level of cancer

Correspondence to: Dr. Ying Yuan, Department of Medical Oncology, The Second Affiliated Hospital, Zhejiang University School of Medicine, Hangzhou 310009, Zhejiang Province,

China

E-mail: yuanying1999@zju.edu.cn

Received: February 26, 2019; Revised: July 08, 2019;

Accepted: July 20, 2019 antigen-199 (CA-199), cancer antigen-153 (CA-153), cancer antigen-125 (CA-125) and carcinoembryonic antigen (CEA) obviously elevated. The levels were 279.7 unit $/ \mathrm{ml}$ (normal level $<37$ unit $/ \mathrm{ml}$ ), 122.2 unit $/ \mathrm{ml}$ (normal level: $<30$ unit $/ \mathrm{ml}$ ), 75.53 unit $/ \mathrm{ml}$ (normal level: $<35$ unit $/ \mathrm{ml}$ ) and 161.97 nanogram $/ \mathrm{ml}$ (normal level: $<5$ nanogram $/ \mathrm{ml}$ ), respectively. Gastroscopy suggested gastric ulcer, and colonoscopy was negative. Enhanced computed tomography (CT) of abdomen and pelvic cavity revealed multiple patchy high-density shadows in centrum, pelvis and proximal femur. Positron emission tomography/ computed tomography (PET/CT) (Figure 1) suggested extensive bone destruction with increased radioactive intake and maximum standard uptake value (SUV) of 14.1. It also showed a mass with a slight increase in radioactive intake (SUV of 3.3) partially obstructing the bronchial cavity in the lower lobe of left lung. Although CA-199 and CA-125 were elevated in this patient, enhanced CT of abdomen and PET/CT showed no evidence of pancreatic/ovarian metastasis or primary pancreatic/ovarian cancers. Chest CT enhancement scan (Figure $2 \mathrm{~A}$ ) revealed blocked bronchial cavity in the lower lobe of left lung with pulmonary atelectasis, and a small amount of effusion in the left thoracic cavity. The patient refused to undergo bronchoscopy. We performed CT-guided biopsy of the right ilium and sacrum. Pathological results showed metastatic adenocarcinoma. Combined with immuno-histochemicalo results, CK (AE1/AE3) +++, CK7 +++, CK20 -, CDX2 -, TTF-1 +++, NapsinA +++, Ki-67 30\%+, P53 +), the patient was eventually diagnosed with lung adenocarcinoma with multiple bone metastasis. NGS of 168 cancer-related 


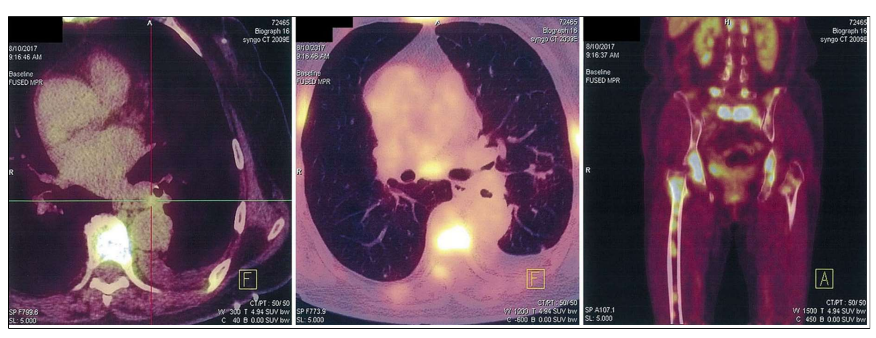

Figure 1: Positron emission tomography/computed tomography (PET/CT).

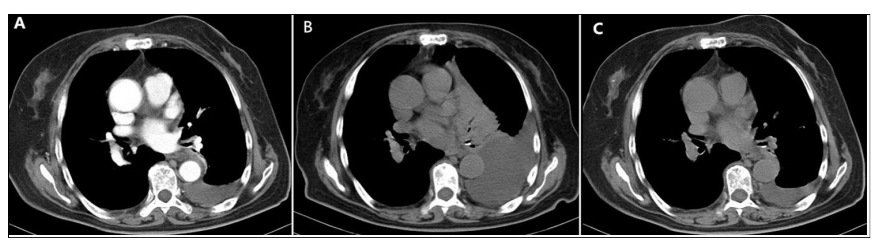

Figure 2: (A) Chest contrast enhanced CT before afatinib; (B) Thoracic highresolution CT after afatinib; (C) Thoracic high-resolution CT after gefitinib.

Table I: EGFR mutation subtype occurring in exon 18-21 and their frequency.

\begin{tabular}{lc}
\hline EGFR mutation subtype & Frequency (\%) \\
\hline Common mutations & 48 \\
Exon 19 deletion & 43 \\
Exon 21 point mutation (L858R) & \\
\hline Uncommon mutations & $3-4$ \\
Exon 18 point mutation (G719X, E709X) & 0.5 \\
Exon 19 insertion & $10(1-17)$ \\
Exon 20 insertion & 1 \\
Exon 20 point mutation (S768I) & 1 (inherited) \\
Exon 20 point mutation (T790M) & 2 \\
Exon 21 point mutation (L861Q)
\end{tabular}

EGFR: Epidermal growth factor receptor.

genes (Burning Rock Biotech, Guangzhou, People's Republic of China) was performed on DNA derived from the biopsy specimen and circulating tumor DNA (ctDNA) derived from whole blood sample. Both showed the patient harboured a mutation (NM_005228.3, c.2217 2234dup) in the EGFR gene exon 19, resulting in a six amino acids insertion (p.Lys745_ Glu746inslleProValAlalleLys). She took afatinib at a starting dose of $40 \mathrm{mg} /$ day from September 10, 2017 and showed great improvement in her pain and other symptoms.

However, about one month later, thoracic high-resolution scanning CT (Figure $2 \mathrm{~B}$ ) revealed solid mass beside the left parahilar enlarged and the left pleural effusion increased compared to the images taking before afatinib. The patient presented symptoms of nausea, vomiting, asthenia and anorexia. Limited effectiveness of afatinib was observed in our patient. We recommended the patient for the genetic test of EGFR T790M. However, because of the fact that the third-generation TKI (AZD9291) was not been included in the coverage of the Chinese healthcare insurance system in October, 2017, patients ought to pay about RMB 50,000 (about 7,500 dollars) a month for taking the third-generation TKI. This was an unaffordable price for our patient. Thus, the patient refused to take genetic test. Moreover, the patient also refused to take chemotherapy. Taking into account the economic situation of the patient and effectiveness of gefitinib in patients with EGFR exon 19 insertion mutation of previous literature, we decided to try the first generation TKI, which had already been included in the coverage of the Chinese healthcare insurance system and the cost of medicine was only about RMB 1,000 (150 dollars) per month. So gefitinib was started at a standard dose of $250 \mathrm{mg} /$ day from October 16, 2017. Thoracic high-resolution scanning CT on October 31, 2017 (Figure 2C) showed that the left pleural effusion was obviously resolved. From November 25, 2017, the patient began to be unconscious, incontinent of urine and feces. Finally, the patient died on December 20, 2017 and her overall survival (OS) was 133 days (4.4 months).

\section{DISCUSSION}

Activating EGFR mutations are main oncogenic drivers in NSCLC. In Asian NSCLC patients, EGFR mutations are detected in about $40-60 \%$ of cases. ${ }^{4}$ EGFR mutations can be divided into two categories: common mutations and uncommon mutation. Exon 19 deletions and exon 21 point mutation (L858R) are the two most common mutations, which also called classical mutations. They account for approximately $48 \%$ and $43 \%$ of all EGFR mutations, respectively. The remaining $10 \%$ of EGFR mutations are reported to occur in exon 18-21 and their subtype and frequency are listed in Table I.5-7 However, due to the complexity of uncommon mutations and the lack of large cohort studies, treatment aiming at EGFR uncommon mutations still remains a big challenge.

Exon 19 insertions are one subtype of the rare EGFR mutations. Park et al. reported two of three cases with exon 19 insertions responding to gefitinib, but time to progression (TTP) was relatively short (mean, 5.9 months). ${ }^{8}$ lyevleva et al. reported three patients with exon 19 insertions receiving gefitinib and TTP were 5 months, 9 months, and 11 months. ${ }^{9}$ Lin et al. also reported three patients received EGFR-TKIs (two gefitinib, one erlotinib) and their progression-free survival (PFS) were 5 months, 22 months and 2 months, respectively. $10 \mathrm{He}$ et al. reported three of four patients harbouring exon 19 insertions sensitive to erlotinib or afatinib (TTP: 14 months, 19 months and 50 months) and they suggested that patients with exon 19 insertions should be treated with EGFR-TKs. ${ }^{2}$

In our patient, we found an 18-base pair insertion between the $2217^{\text {th }}$ and 2234th nucleotides in EGFR exon 19 , which lead to a six residue insertion in the protein structure between codon 745 and codon 746 (p.Lys745_Glu746inslleProValAlalleLys). According to the published literature, all the reported EGFR exon 19 insertion subtypes are non-frame shift mutations. Except 
for one patient, who had 15-base pair insertion, to all other had 18-base pair insertion.2,10 In addition, existing literature shows insertion types to include KIPVAI, IPVAIK, VPVAIK and TPVAIK2, whereas our patient had IPVAIL insertion, a new type never reported before. Its response to EGFR-TKIs treatment was less than satisfactory. Our patient's overall survival was only 133 days (4.4 months).

Despite the limitations of a case report, the present case demonstrates that patients harbouring this rare subtype of EGFR exon 19 insertion mutation may have a poor prognosis. Further experiences are required to characterise these rare mutations.

\section{PATIENT'S CONSENT:}

Written informed consents for publication of this case report and accompanying images were obtained both from the patient during her lifetime and from the patient's daughter after the patient died.

\section{CONFLICT OF INTEREST:}

Authors declared no conflict of interest.

\section{AUTHORS' CONTRIBUTION:}

NZ: Contributed to the conception of the work, the acquisition of data for the work and the writing of the manuscript.

CD: Contributed to the design of the work, the interpretation of the results and the revising the manuscript.

SW: Contributed to the interpretation of the results and the revising the manuscript.

YY: Contributed to the conception of the work, the interpretation of results for the work and the revising the manuscript.

\section{REFERENCES}

1. Herbst RS, Heymach JV, Lippman SM. Lung cancer. N Engl J Med 2008; 359:1367-80.

2. He M, Capelletti M, Nafa K, Yun $\mathrm{CH}$, Arcila ME, Miller VA, et al. EGFR exon 19 insertions: A new family of sensitizing EGFR mutations in lung adenocarcinoma. Clin Cancer Res 2012; 18:1790-7.

3. Oken MM, Creech RH, Tormey DC, Horton J, Davis TE, McFadden ET, et al. Toxicity and response criteria of the Eastern Cooperative Oncology Group. Am J Clin Oncol 1982; 5:649-55.

4. Peng Y, Cui H, Liu Z, Liu D, Liu F, Song Y, et al. Apatinib to combat EGFR-TKI resistance in an advanced non-small cell lung cancer patient with unknown EGFR status: A case report. Onco Targets Ther 2017; 10:2289-95.

5. Karachaliou N, Molina-Vila MA, Rosell R. The impact of rare EGFR mutations on the treatment response of patients with nonsmall cell lung cancer. Expert Rev Respir Med 2015; 9:241-4.

6. O'Kane GM, Bradbury PA, Feld R, Leighl NB, Liu G, Pisters KM, et al. Uncommon EGFR mutations in advanced non-small cell lung cancer. Lung Cancer 2017; 109:137-44.

7. Castellanos E, Feld E, Horn L. Driven by mutations: The predictive value of mutation subtype in EGFR-mutated nonsmall cell lung cancer. J Thorac Oncol 2017; 12:612-23.

8. Park J, Kondo C, Shimizu J, Horio Y, Yoshida K, Hida T. EGFR exon 19 insertions show good response to gefitinib, but short time to progression in Japanese patients. J Thorac Oncol 2014; 9:e10-1.

9. Iyevleva AG, Mitiushkina NV, Karaseva NA, Orlov SV, Volodina LN, Kulikova YE, et al. Lung carcinomas with EGFR exon 19 insertions are sensitive to gefitinib treatment. $J$ Thorac Oncol 2014; 9:e31-3.

10. Lin YT, Liu YN, Wu SG, Yang JC, Shih JY. Epidermal growth factor receptor tyrosine kinase inhibitor-sensitive exon 19 insertion and exon 20 insertion in patients with advanced nonsmall-cell lung cancer. Clin Lung Cancer 2017; 18:324-32 e1. 